\title{
The Experimental Platform of Frosting by Vapor on Cryogenic Surface in Vacuum Environment
}

\author{
Guo Qinliang ${ }^{1, a}$, Qie Dianfu ${ }^{1}$, Shang Yonghong ${ }^{1}$, Wang Jing ${ }^{1}$ and Bi Yanqiang ${ }^{1}$ \\ ${ }^{1}$ Beijing Institute of Spacecraft Environment Engineering, China
}

\begin{abstract}
This paper introduces an experimental platform for studying frosting on the cryogenic surface in vacuum environment. This platform contains vacuum system, cold surface temperature control system, image acquisition system, data acquisition system and vapor generation device. The cold surface temperature can be varied in the range of -185 to $0^{\circ} \mathrm{C}$, the vacuum degree of the chamber can be controlled in the range of 1 pa to $1 \mathrm{bar}$, and the flow rate of water vapour can be controlled accurately. The experimental platform can be used to study the micro-morphology and the thermal conductivity of frost layer.
\end{abstract}

\section{Introduction}

Frosting is a common phenomenon in nature, and it also exists in the engineering field of cryogenics, refrigeration and aerospace. When the cold surface temperature is lower than the freezing point temperature of the water vapor, the water vapor will frost on the cold surface. At present, in the field of aerospace, manned spacecraft has not realized the closed-loop water circulation yet. It will discharge a certain amount of water into the space in orbit. Water sublimator of space suit also releases some water vapor. The released vapor will be around the spacecraft, then it maybe frost on the cold surface of the spacecraft. It will have a bad impact on the spacecraft performance. For example, frosting on the radiating surface may affect the thermal control performance of spacecraft; frosting on the lens may significantly influence the camera optical properties; frosting on the key joint may affect the moving performance of the mechanism.

The phenomenon of frosting also exists in the spacecraft ground simulation test. There is inevitable a certain amount of water vapor inside the spacecraft simulation chamber, which will deposited a layer of frost on the cryogenic surface. If the frost is formed on the critical surface of the test piece, it will affect the original performance of the test piece, resulting in the inaccuracy of the test result and even the failure of the whole test.

This paper is aimed to build experimental platform of frosting in vacuum cryogenic environment for studying the micro-morphology and the thermal conductivity of frost layer. It has an important engineering value for the safe operation of the spacecraft. There are some studies on the topography of the frost in vacuum cryogenic environment. For ultra-low cold surface temperatures, the frost layer growth is controlled by the outer boundary layer mechanism. There is a tendency that the frost layer thickness decreases as the cold surface temperature decreases, which is totally different to the frost growth on the cold surfaces of ordinary-low temperature[1]. Xiaomin $\mathrm{Wu}[2,3]$ studied the frost

a Corresponding author: 1159497408@qq.com 
formation on aluminum surface with grooved structures, and concluded that the frost prefers to grow at the top edges and corners of the structures instead of inside the grooves on the grooved surfaces. Boxe etc[4],measured the surface grain size and distribution, specific surface area, density, porosity of the frost under the pressure at $260 \mathrm{~Pa}$ by using electronic microscope. The measured porosity of the frost layer is 0.31. Laufer[5] studied the frost film condensed by pure water vapor at $100 \mathrm{~K}$ temperature, and found that the frost film had a needle structure. As the heating continued, it remained until the ice film completely disappeared. Mayer and Atteberry[6,7] found a similar needle-like structure on the surface of the frost film. Mayer[7] concluded that there were a large number of microscopic holes in the frost film, and the micropores provided a large amount of adsorption ratio (a few hundred $\mathrm{m}^{2} / \mathrm{g}$ ). As the ice film grew, the adsorption ratio decreased gradually. Schmitt[8] came to the similar conclusion that micro-pores are only a few microns in size. The effective thermal conductivity of porous media depends on the thermal conductivity of each phase and the porosity, and on the array formed by particles and pores[9,10]. Zou Tonghua [11] studied the frost deposited on the surface of the cold well coils of the freeze dryer with pure ice as the freeze-dried material. In his experiment, the cold trap temperature is $-40^{\circ} \mathrm{C}$, the vacuum degree is lower than $10 \mathrm{~Pa}$. Thermal conductivity of the frost layer was obtained by Fourier Law within the range of 0.15 to $0.25 \mathrm{w} /(\mathrm{m} \cdot \mathrm{K})$, that is considered to be $1 / 4$ to $1 / 3$ at the atmospheric pressure.

At present, there is less research on the frosting phenomenon in the vacuum environment, lack of detail image information of the microstructure of the frost layer. The variation range of the thermal conductivity of the frost layer is large, and the data comparison verification is lacking. Therefore, it is necessary to establish an experimental platform to study frost phenomenon in the vacuum cryogenic environment. Base on the platform, temperature data and morphology image of the frost will be obtained, and it will lay the foundation for the research in the future.

\section{Experimental apparatus}

The vacuum cryogenic experimental platform is shown in figure 1, the experimental system consists of five parts: cold surface temperature control system, image acquisition system, data acquisition system and vapor generation device.

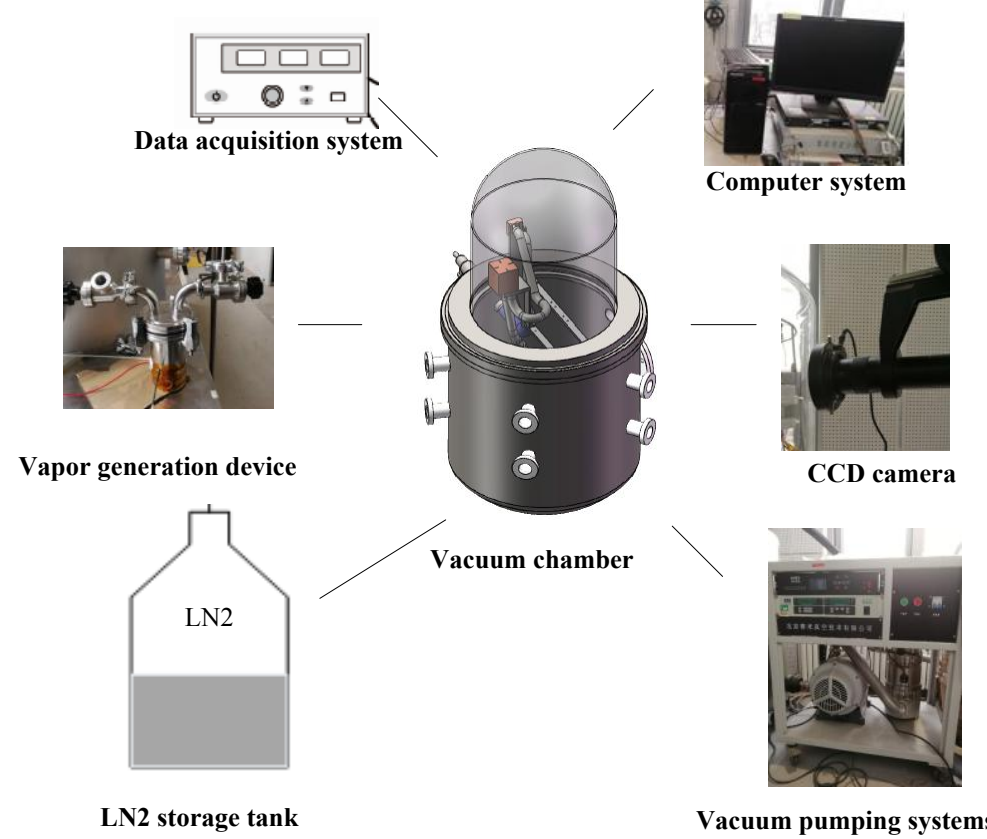

Figure 1. Schematic of the experimental platform 


\subsection{Vacuum system}

The vacuum system mainly consists of vacuum chamber and vacuum pumping system. The vacuum chamber contains two parts: a stainless steel base and a quartz glass bell jar, which are connected by a flange. The effective volume of the chamber is $\Phi 380 \times 800 \mathrm{~mm}$. The quartz glass has such a good optical permeability that we can observe frost from outside the chamber by the microscopic camera. The vacuum pumping system is composed of dry pump and molecular pump, and the ultimate pressure is $5 \times 10^{-3} \mathrm{~Pa}$. The vacuum degree can be controlled by the pumping rate and the outgassing rate. Figure 2 shows the vacuum degree changes during an experiment.

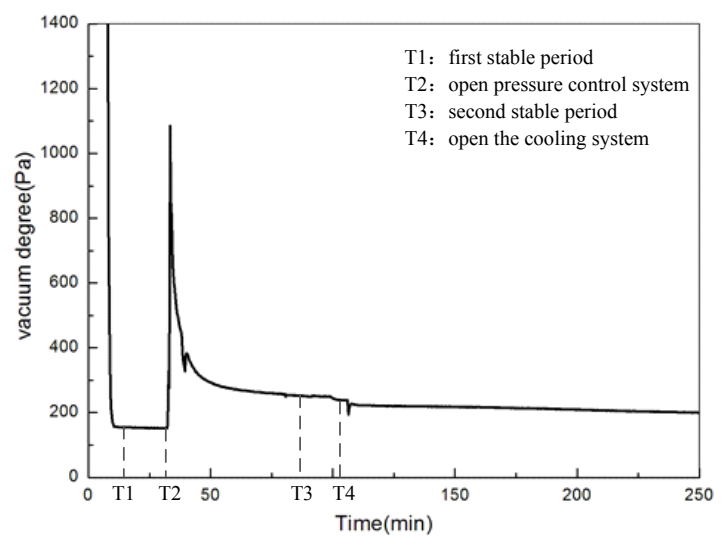

Figure 2. Vacuum degree as a function of time

\subsection{Cold surface temperature control system}

The surface temperature of the cold plate is controlled by the cooling system and the heating system. The cooling system has an open structure: liquid nitrogen flows out of the dewar, enters into cooling box to exchange heat with copper block, flows through cryogenic valve, is discharged into the atmosphere. The LN2 flux can be precisely controlled by the valve. The heating system consists of heater and program-controlled power. The cooling system and the heating system are regulated by a PID controller. By this way, the cold surface temperature can be controlled ranged from -185 to $0^{\circ} \mathrm{C}$ with the fluctuating range of $\pm 0.3^{\circ} \mathrm{C}$. The cold surface temperature in the experiment is shown in figure 3(a), 3(b) shows a locally amplified curve in steady state condition.

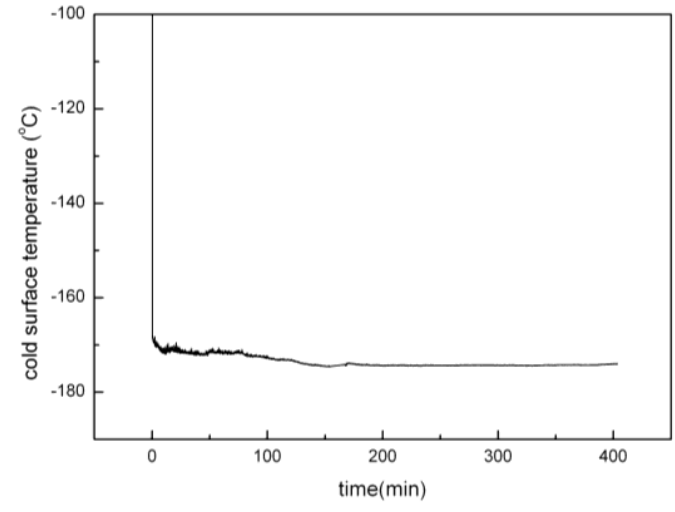

(a)

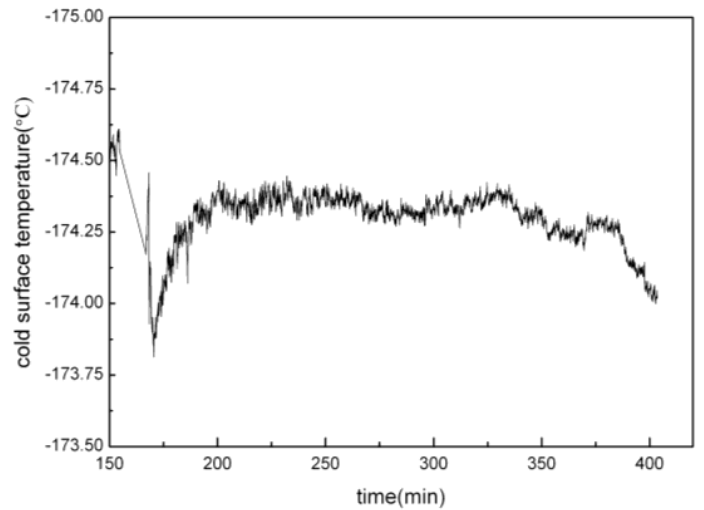

(b)

Figure 3. Cold surface temperature as a function of time.(a)overall (b) detail view 
In this experiment, water vapor was generated by ice sublimation under low pressure. The vapor generation device is shown in figure4. Before the experiment, the pure distilled water is frozen into ice, then the prepared ice is added to the ice storage. The outer wall of the storage room is heated by the heater. After the experiment began, the ice began to sublimate as the pressure in the reservoir decreased, and the sublimation latent heat was provided by the heater, so that the water vapor could be produced continuously. The flow of vapor was controlled by the hand valve installed on the transmission line. The effect of wind speed on the experiment is not considered.

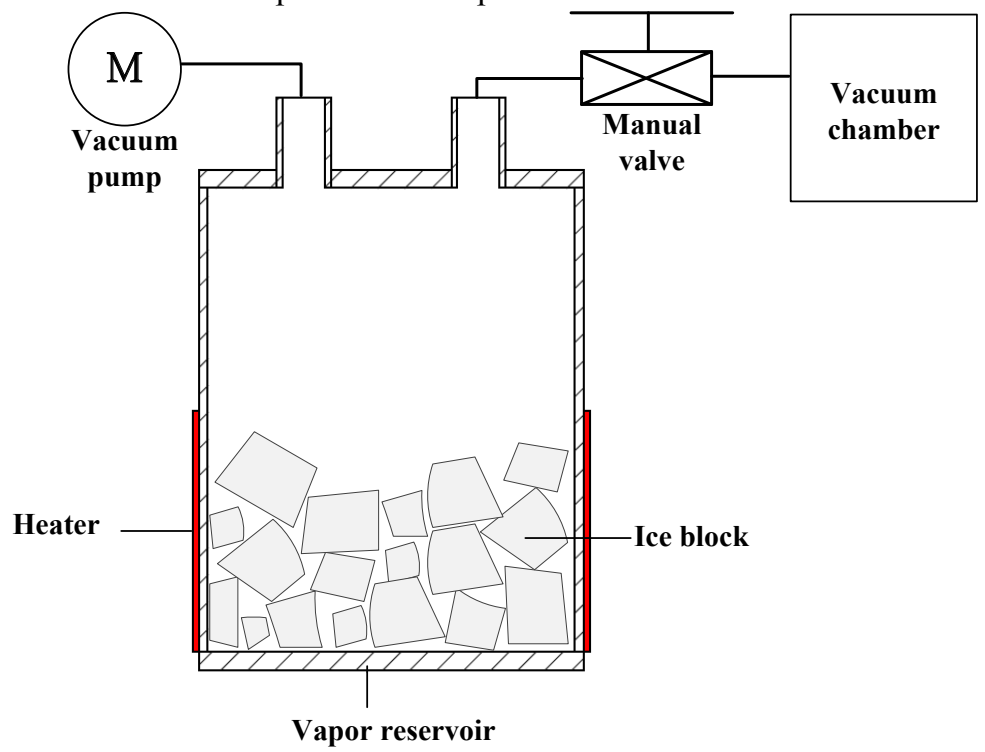

Figure 4. Schematic of the vapor generation device

\subsection{Image acquisition system}

The image acquisition system is used to measure the thickness of the frost layer and observe the morphology of the frost. The system consists of computer, image capture card, microscope and CCD camera (shown in figure5). The microscope is produced by Bo Hong optical instrument factory (the optical magnification can reach 4.5 times). The experiment uses the critical illumination for better observation. The light source has enough luminance to ensure the brightness of the image with no distortion on the frost surface temperature. The frosting image can be transported to the computer by the combination of photoelectric conversion and upper computer program. The change of frost can be recorded by the image capture card and be real-time monitored on the display.

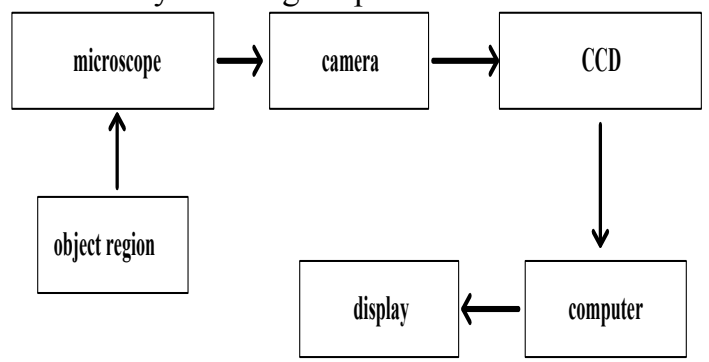

Figure 5. Schematic of the image acquisition system

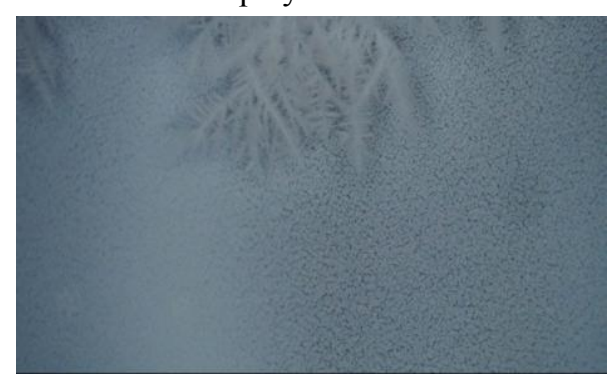

Figure 6. Image of frost structure by CCD camera

In the experiment, the camera has two directions: the horizontal one is used to observe the thickness and structure of the frost while the vertical one is used to observe the distribution of preliminary frost crystal formed on the cold surface (Shown in figure 6). 


\subsection{Data acquisition system}

The cold plate, whose material is T2-copper, is shown in figure7. The plate is 70 millimeters in height, which are divided into two parts. The upper part of the cold plate, that is $50 \mathrm{~mm}$ height, is the copper block. The top surface is used to frost, and the rest surfaces are arranged for the heating plates whose outside surfaces are covered with $10 \mathrm{~mm}$ thick PTFE insulation board to reduce heat leakage.

There are four thermocouples inserted into the holes to measure the top surface temperature, the other four thermocouples to measure the temperature of the block at the position of $20 \mathrm{~mm}$ below the top surface. The lower part of the cold plate is the cold box, in which liquid nitrogen evaporate and cool the upper copper block.

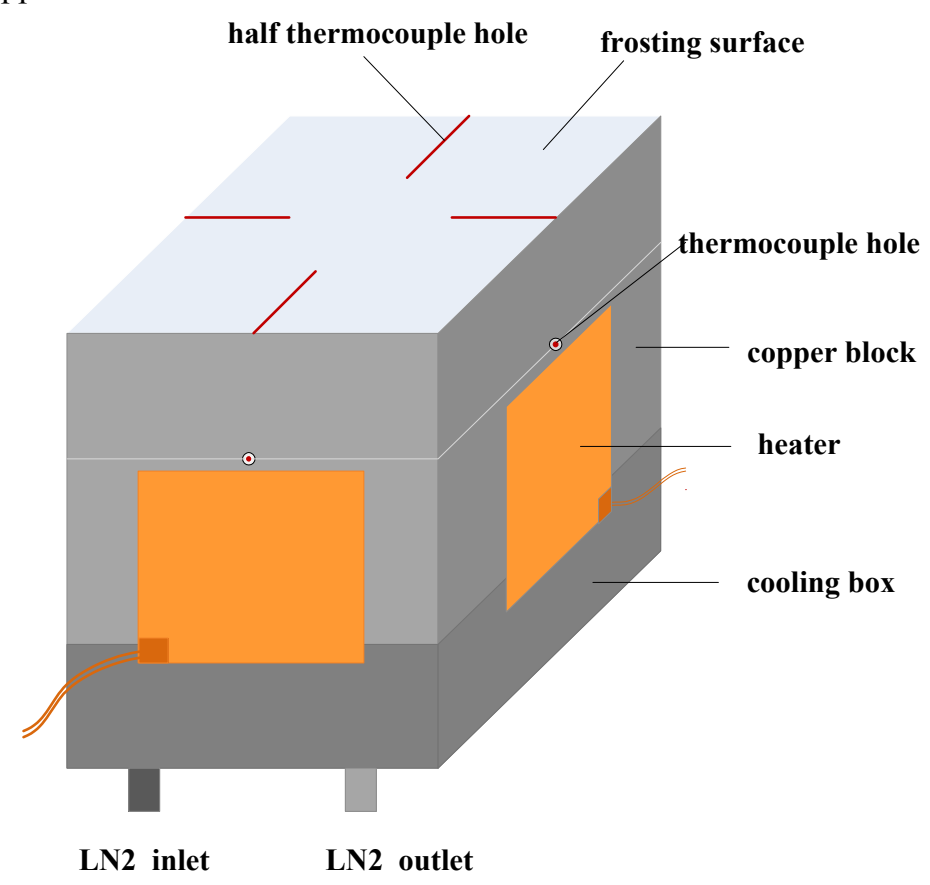

Figure 7. Structure diagram of the cold plate

From the above, we can obtain the temperature of the copper block, the top surface and the frost layer. Based on Fourier's law, the thermal conductivity of the frost can be calculated with the copper thermal conductivity known.

\section{Conclusions}

This paper introduced the principle and structure of the vacuum cryogenic experimental platform in detail. This experimental platform is aimed to study water vapor desublimation phenomenon. Through the test, the experiment conditions, including the cold surface temperature, the vacuum and the flow rate of water vapor, can be controlled accurately. The measurement system has the ability to observe the morphology of the frost and measure the thermal conductivity of the frost.

\section{Acknowledgement}

This work was supported by the National Natural Science Foundation of China (No.51506009).

\section{References}

1. L Li, Z Liu, Y Li, Y Dong. Frost deposition on a horizontal cryogenic surface in free convection[J]. International Journal of Heat and Mass Transfer,113(2017):166-175.

2. Xiaomin $\mathrm{Wu}$, Fuqiang Chu, Qiang Ma, Bei Zhu. Frost formation and frost meltwater drinage characteristics on aluminum surface with grooved structures[J]. Applied Thermal Engineering, 118(2017),448-454. 
3. Xiaomin $\mathrm{Wu}$, Fuqiang $\mathrm{Chu}$, Qiang Ma. Frosting model based on phase change driving force[J]. International Journal of Heat and Mass Transfer,110(2017):760-767.

4. C.S. Boxe, B.R. Bodsgard, W. Smythe, M.T. Leu. Grain sizes, surface areas, and porosities of vapor-deposited $\mathrm{H} 2 \mathrm{O}$ ices used to simulate planetary icy surfaces. Journal of Colloid and Interface Science 309 (2007) 412-418

5. Laufer, D., Kochavi, E., Bar-Nun, A., 1987. Phys. Rev. B 36, 9219-9227.

6. Mayer, E., Pletzer, R., 1986. Nature 319, 298-301.

7. Atteberry, C., 1999. MS. Thesis, University of Virginia.

8. Schmitt, B., Ocampo, J., Klinger, J., 1987. J. Phys. (Paris) 48, C1,519-525.

9. Silvia Negrelli, Valter S. Nascimento Jr.A study of the effective thermal conductivity of frost formed on parallel plate channels[J]. Experimental Thermal and Fluid Science,78(2016), 301-308.

10. S. Negrell, C.J. Hermes, A semi-empirical correlation for the thermal conductivity of frost[J]. International Journal of Refrigeration,58(2015),243-252.

11. ZOU T H, XUE Y L, SUN Y. Experimental study on performance of frost on cold-trap used by vacuum freeze-drying machine[J]. Fluid Machinery,2009,37(4). 